\title{
MEMS gas flow sensor based on thermally induced cantilever resonance frequency shift
}

\author{
Robert Blue, James G. Brown, Lijie Li, Senior Member, IEEE, Ralf Bauer, Member, IEEE, and Deepak \\ Uttamchandani, Fellow, IEEE
}

\begin{abstract}
This paper reports a novel MEMS gas flow sensor that relies on the temperature drop induced when the gas flows over an electrically heated MEMS triple-beam resonator. Modelling, simulation and characterization of the sensor has been undertaken to quantify the temperature-induced shift of resonance frequency of the resonator, which can be directly related to the rate of gas flow over the heated resonator. The MEMS resonator was actuated into mechanical resonance through application of an AC voltage to an aluminum nitride (AIN) piezoelectric layer coated on the central beam of the triplebeam resonator. A reversible change in resonance frequency was measured experimentally for nitrogen flow rates up to 5000 $\mathrm{ml} / \mathrm{min}$. At $5 \mathrm{~V}$ operating voltage the linear response fit measured from experiments yielded a $67 \mathrm{ml} / \mathrm{min}$ per $\mathrm{Hz}$ slope over a flow rate range from $0 \mathrm{ml} / \mathrm{min}$ to $4000 \mathrm{ml} / \mathrm{min}$.
\end{abstract}

Index Terms-Anemometer, Cantilever, Electrothermal, Micromechanical systems (MEMS), Piezoelectric, Resonance.

\section{INTRODUCTION}

$\mathrm{M}$ ICROELECTROMECHANICAL systems (MEMS) based sensors offer significant advantages of high-sensitivity, small size, low power consumption, and low unit cost via highvolume manufacturing [1]. Consequently, MEMS sensors are increasingly replacing traditional measurement devices for many sensing applications, and enable integration of novel sensors in new application areas [2]-[4]. One versatile sensing approach is based on utilizing MEMS resonators to make microsensors ranging from accelerometers to sensors targeting specific biological or chemical species [5]. This approach operates by detecting changes of resonance frequencies of mainly silicon-based micromechanical structures. These changes can be detected using optical, capacitive, piezoresistive or piezoelectric sensing mechanisms. A MEMS resonator incorporating a piezoelectric material, for example, can directly convert electrical to mechanical motion (and vice versa) [6] allowing direct integration on-chip with CMOS technology to form a miniaturized sensor which can be interrogated using wireless communication [7].

The approach of detecting resonance frequency changes can be used for determining the velocity of gas flow around a MEMS cantilever. Accurate determination of the velocity of

R. Blue, J. G. Brown, R. Bauer and D. Uttamchandani are with the Centre for Microsystems and Photonics, University of Strathclyde, G1 1XW, United Kingdom (e-mail: d.uttamchandani@ strath.ac.uk). gas flows is important in diverse areas such as weather forecasting, pollution monitoring, industrial process control and medical devices [8]. Traditionally this measurement has been accomplished using either a bulk mechanical or thermal flow sensors. Bulk sensors use a rotating section whose rotational speed is dependent on the gas flow speed [9], whilst thermal flow sensors use the change in electrical resistance of a heated metal with temperature, which can be directly related to the velocity of surrounding gas via its cooling effect [10].

A heated silicon MEMS resonator will have a natural resonance governed by the design, dimensions, temperature and material properties of the resonator [11], [12]. If the device is actuated at its resonance frequency (for a fixed actuation voltage) a change in the velocity of a gas flow around the heated resonator will modify its temperature and will induce a change in the natural resonance frequency, accompanied by a change in the maximum displacement and/or velocity of the resonator. Change of resonance frequency with temperature of MEMS resonators is a well-known effect that has been used for temperature sensing [13]. Hence, measurements of either the resonance frequency or maximum displacement can be directly related to the rate of gas flow over the resonator. A gas flow sensor using a resonating, heated MEMS microbridge (fixed at both ends) and with embedded piezoresistors has previously been reported [14], while free-standing MEMS cantilevers have also previously been used to determine gas flow, primarily using deflection-based (i.e., not frequency-based) sensing of the static microstructures [15], [16].

In this paper, we report a frequency output MEMS based gas flow sensor that uses a combination of piezoelectric actuation and electrothermal heating to operate in a heated resonating configuration. The sensor design employs a triple-beam cantilever, which is adapted from an electrothermal actuator design used as a component in optical MEMS devices that have been employed for imaging [17] and laser control purposes [18]. The sensor uses thermally-induced changes of the resonance frequency of the silicon triple-beam cantilever structure to measure gas flow. Gas flow over the triple-beam resonator causes convective heat loss, leading to a change in temperature and therefore a related change in resonance frequency that is measured using optical techniques. Compared to the previous research describing a heat-loss based MEMS

L. Li is with the Multidisciplinary Nanotechnology Centre, College of Engineering, Swansea University, Swansea SA1 8EN, United Kingdom (email: L.Li@Swansea.ac.uk). 
resonator flow-sensor, which was based on electothermally generated oscillations in a double-fixed beam architecture [14], our device: (a) uses an outer U-shaped cantilever which allows greater heat loss; (b) requires lower drive voltage to generate oscillations; and (c) uses aluminum nitride (AlN) as the piezoelectric material to generate oscillations which is more electrically efficient, and also has greater compatibility with MEMS fabrication processes compared with PZT, so is a more practical piezoelectric material in the MEMS manufacturing context.

\section{MEMS SENSOR DESIGN}

The MEMS gas flow sensor with frequency output uses a Ushaped folded outer beam, consisting of two cantilever beams joined at their free ends by a cross connecting beam, as the heated element within the device. A DC current is passed through the folded beam ('hot' beam) to raise its temperature. A central cantilever 'cold' beam is mechanically connected to the folded 'hot' beam, but without current flow and related active heating. The central beam, with its AlN piezoelectric layer coating, generates the oscillations of the overall structure. Figure 1 shows a top view and side view schematic of the MEMS cantilever-based gas flow sensor. The triple-beam structure consists of three $1800 \mu \mathrm{m}$ long, $50 \mu \mathrm{m}$ wide and 10 $\mu \mathrm{m}$ thick p-doped single crystal silicon beams which are connected via a $80 \mu \mathrm{m}$ wide, $10 \mu \mathrm{m}$ thick and $390 \mu \mathrm{m}$ long common connection. The outer beams, used for DC electrothermal heating of the device, consist of only a doped silicon device layer. The central beam generates the device resonant movement being driven by a piezoelectric layer on top of the Si device layer, the latter of which also acts as a bottom electrode. The piezoelectric layer structure consists of a $500 \mathrm{~nm}$ thick AlN piezoelectric film on which a $1 \mu \mathrm{m}$ thick Al top electrode is deposited for piezoelectric actuation in the $d_{31}$ mode. On the fixed side, the electrical contact layers are electrically isolated using a $1 \mu \mathrm{m}$ oxide layer. The device is fabricated using a commercial multi-user fabrication process offered by MEMSCAP Inc. (PiezoMUMPs). The triple-beam geometry is fully backside released and therefore allows airflow both through the device as well as over it.

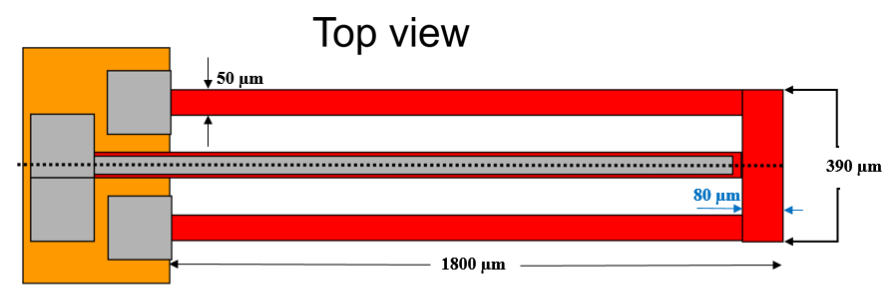

Side view

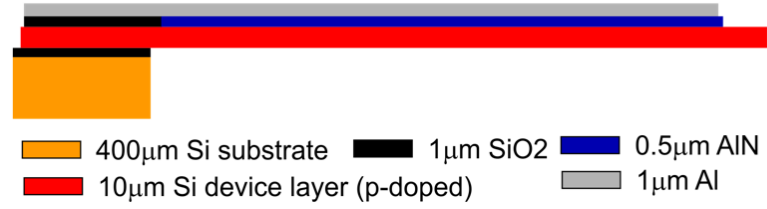

Fig. 1. Top and side views of device structure and material layers forming a MEMS piezo-coated cantilever sensor.

\section{Modelling OF GAS Flow Resonator SENSOR}

Initially, the eigenfrequencies of the MEMS triple-beam resonator were simulated using COMSOL Multiphysics 4.2a (COMSOL Ltd., UK), taking into consideration initial stresses present in the silicon device layer due to fabrication, which leads to an initial out of plane bending of the free end of the connected cantilever beams. This predicted a fundamental flexural resonance frequency around $3.70 \mathrm{kHz}$ for the overall structure with piezoelectric coating, with higher order modes at $23.55 \mathrm{kHz}$ and $29.20 \mathrm{kHz}$ respectively (shown in Figure 2).

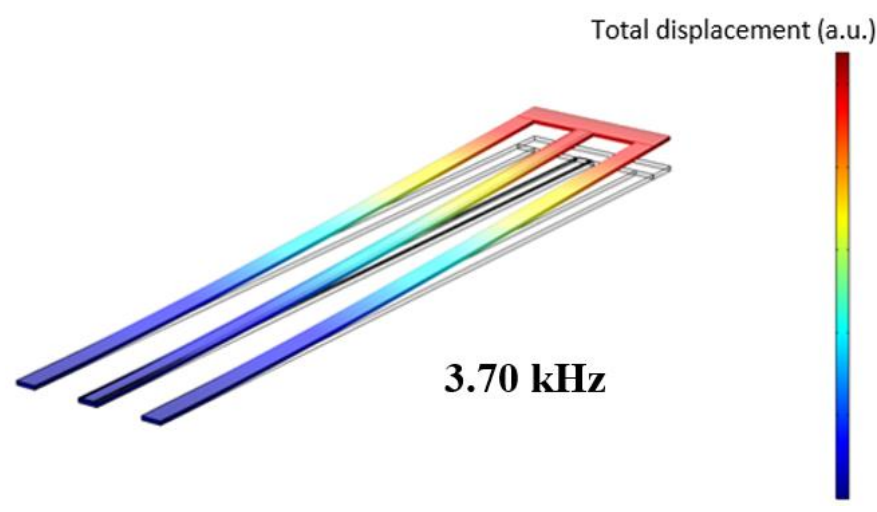

(a)

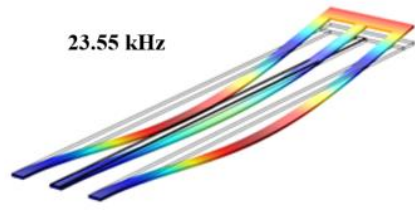

(b)

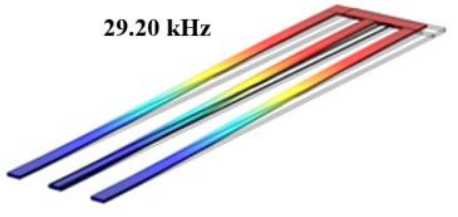

(c)
Fig. 2. Simulated resonance frequencies of proposed MEMS piezo-coated cantilever sensor. (a) Out-of-plane mode 1: $3.70 \mathrm{kHz}$ (b) Out-of-plane mode 2: $23.55 \mathrm{kHz}$ (c) In-plane mode 3: $29.20 \mathrm{kHz}$.

To model the behavior of the heated resonator as a gas flow sensor, a mathematical model was built describing electrothermal, gas flow-temperature, and temperatureresonant frequency processes. A schematic of the modelled structure is shown in Figure 3(a). First, an existing electrothermal model [19] was used to calculate the temperature distribution of the silicon folded beam structure under DC electrical voltage applied between the electrodes of the structure. This process is governed mathematically by two second order partial differential equations, namely sourced (heated) and unsourced, shown in equations (1) and (2) respectively.

$$
\begin{aligned}
& k_{T} T_{x_{1}}^{h} A \frac{\partial^{2} T_{x_{1}}^{h}}{\partial x_{1}^{2}}=-\frac{A V^{2}(t)}{\rho_{s} T_{x_{1}}^{h} L_{h}^{2}}+\frac{k_{\text {air }}}{\Delta z}\left(T_{x_{1}}^{h}-T_{a}\right)+ \\
& P h_{c}\left(T_{x_{1}}^{h}-T_{a}\right)-P \varepsilon \sigma_{0}\left(T_{x_{1}}^{h}\right)^{4}
\end{aligned}
$$


$k_{T} T_{x_{2}}^{c} A \frac{\partial^{2} T_{x_{2}}^{c}}{\partial x_{2}^{2}}=\frac{k_{a i r}}{\Delta z}\left(T_{x_{2}}^{c}-T_{a}\right)+P h_{c}\left(T_{x_{2}}^{c}-T_{a}\right)-$ $P \varepsilon \sigma_{0}\left(T_{x_{2}}^{c}\right)^{4}$
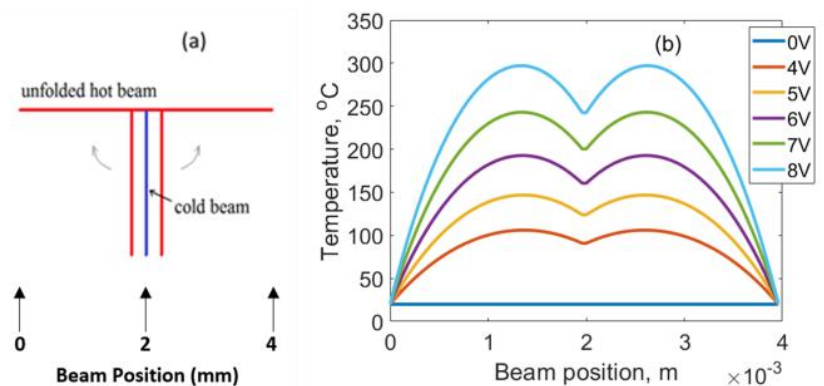

Fig. 3. (a) Schematic used in the calculation. (b) Calculated temperature distribution along the unfolded hot beam for a range of applied voltages.

Equation (1) describes the steady state Joule heating of the outer two connected beams (i.e. the folded beam), while equation (2) represents the central 'cold' beam which is not actively heated. In equation (1), the terms on the right side are input power for the 'hot' beam $(\mathrm{V}(\mathrm{t})$ is the source term), heat conduction to the substrate, convective heat loss, and the radiation loss respectively. There is no input power in equation (2) for the 'cold' beam. The left side of both equations (1) and (2) is the heat conduction through the silicon beam to the anchors, where the room temperature of $293 \mathrm{~K} / 20^{\circ} \mathrm{C}$ is set. Variables and parameters in both the equations are listed in Table 1.

Under various applied DC voltages, the temperature distribution along the unfolded hot beam was calculated and is shown in Figure 3(b). The effective emissivity, also termed as the effective absorptivity, varies from 0.4 to 0.71 for silicon [20], and here we take the value of 0.5 in the modelling. The emissivity value of 0.5 was extracted from experimental measurements using a thermal imaging camera. $h_{c}$ (convective

TABLE I

LIST OF SYMBOLS USED IN THE MODELLING OF THREE-BEAM CANTILEVER

\begin{tabular}{|c|c|c|}
\hline Symbol & Definition & Quantity/Unit \\
\hline $\mathrm{k}_{\mathrm{T}}$ & Thermal conductivity of silicon & Temperature dependent \\
\hline $\mathrm{T}_{\mathrm{x} 1}^{\mathrm{h}}$ & Temperature in the hot beam & ${ }^{\circ} \mathrm{C}$ \\
\hline $\mathrm{T}_{\mathrm{x} 2}^{\mathrm{c}}$ & Temperature in the cold beam & ${ }^{\circ} \mathrm{C}$ \\
\hline $\mathrm{h}_{\mathrm{c}}$ & Air free convection coefficient & Flow rate dependent \\
\hline $\mathrm{x}_{1}$ & Axis of the unfolded hot beam & $\mu \mathrm{m}$ \\
\hline $\mathrm{V}(\mathrm{t})$ & Externally applied voltage & V \\
\hline$\rho_{\mathrm{s}}$ & Resistivity of silicon & Temperature dependent \\
\hline $\mathrm{L}_{\mathrm{h}}$ & Unfolded hot beam & $3900 \mu \mathrm{m}$ \\
\hline $\mathrm{L}_{\mathrm{c}}$ & Cold beam & $1800 \mu \mathrm{m}$ \\
\hline A & Cross-section area of silicon beam & width*thickness \\
\hline $\mathrm{P}$ & Perimeter of the beam & $120 \mu \mathrm{m}$ \\
\hline$\Delta \mathrm{z}$ & $\begin{array}{l}\text { Effective air gap between the device } \\
\text { and the substrate }\end{array}$ & $200 \mu \mathrm{m}$ \\
\hline $\mathrm{k}_{\mathrm{air}}$ & Thermal conductivity of air & $0.026 \mathrm{~W} \mathrm{~m}^{-1} \mathrm{~K}^{-1}$ \\
\hline $\mathrm{T}_{\mathrm{a}}$ & Room temperature & $20^{\circ} \mathrm{C}$ \\
\hline$\varepsilon$ & Effective emissivity of silicon & 0.5 \\
\hline$\sigma_{0}$ & Stephan-Boltzmann constant & $5.6 \times 10^{-8} \mathrm{Wm}^{-2} \mathrm{~K}^{-4}$ \\
\hline
\end{tabular}

coefficient) is taken as 5.7 for free convection, i.e. external air flow rate is $0 \mathrm{~m} / \mathrm{s}$.

The gas flow rate affects the convective coefficient, which induces a temperature drop of the three-beam cantilever. In general, the convective coefficient increases with the air flow rate and there are many empirical equations for this relation. We employ the equation for a flat, small surface [21], which is

$$
h_{c}=5.7+3.8 v
$$

where $h_{c}$ is the convection coefficient and $v$ is the external air flow velocity in $\mathrm{m} / \mathrm{s}$. Most previous empirical equations to calculate the $h_{c}$ are linear as described in ref [21]. It becomes nonlinear $\left(\sim v^{0.5}, \sim v^{0.8}\right)$ for some special flow types such as fully turbulent and mixed flows. Nonlinear equation will cause the average temperature versus $h_{c}$ relation to be nonlinear, leading to a nonlinear frequency/flow rate sensitivity. Substituting the above equation into equations (1) and (2), one can obtain the temperature distribution of the cantilever with different air flow velocities shown in Figure 4, for an applied heating voltage of $5 \mathrm{~V}$. When the air flow rate is $0 \mathrm{~m} / \mathrm{s}$, it indicates that the device is under free convection condition, i.e. no external air flow, as compared with the scenarios where external flows are present.
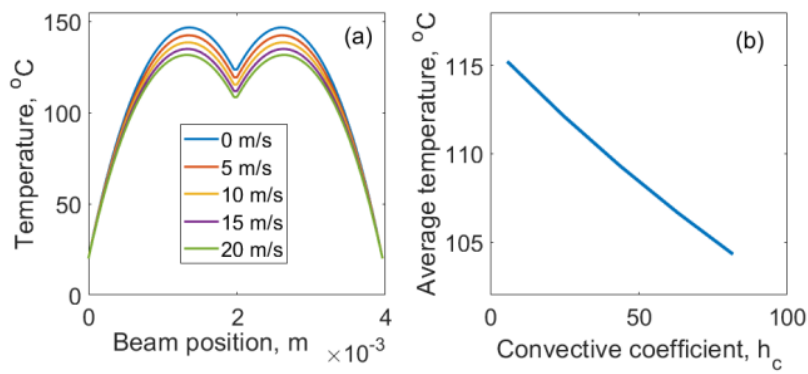

Fig. 4. Calculated beam temperature versus air flow rate at 5 V. (a) Distributed temperature for various flow velocity. (b) Average temperature of the hot beam vs. convective coefficient.

Temperature variation affects the material properties of the mechanical cantilever, mainly the Young's modulus [22], [23]. The effect of the temperature dependent Young's modulus can be simulated using the method described in reference [22]. The analytical expressions for the fundamental resonant frequency $\omega_{1}$ and the temperature dependence of the Young's modulus $E(T)$ of a cantilever are

$$
\begin{aligned}
\omega_{1} & =(1.875)^{2} \frac{t}{L^{2}} \sqrt{\frac{E}{12 \rho}} \\
E(T) & =E_{0}-B T \exp \left(-\frac{T_{0}}{T}\right)
\end{aligned}
$$

where $E_{0}$ is the Young's modulus at room temperature. $B$ and $T_{0}$ are temperature independent constants. The values of $E_{0}, B$, and $T_{0}$ are all taken from the references for silicon as $E_{0}=167.5$ $\mathrm{GPa}, T_{0}=317 \mathrm{~K}$, and $B=15.8 \mathrm{MPa} / \mathrm{K}$ [22]. Calculated relation between the flow rate and average temperature for $5 \mathrm{~V}$ heating 
voltage is shown in Figure 5a. Calculations according to the described procedure have been conducted and the results are shown in Figure 5b. It is shown that the resonant frequency reduces linearly as the temperature increases. We calculated average temperatures for a range of flow rates $(\mathrm{K} /(\mathrm{m} / \mathrm{s}))$, and resonant frequencies for a range of average temperatures from equations (4) and (5) to arrive at resonant frequency versus average temperature $(\mathrm{Hz} / \mathrm{K})$. The combination of the above two relations gives the sensitivity $(\mathrm{Hz} /(\mathrm{m} / \mathrm{s}))$. At $5 \mathrm{~V}$ heating voltage, the calculated sensitivity is $0.085 \mathrm{~Hz} /(\mathrm{m} / \mathrm{s})$. The calculated sensitivity increases with the heating voltage, reaching a value of $0.31 \mathrm{~Hz} /(\mathrm{m} / \mathrm{s})$ for $10 \mathrm{~V}$ applied heating voltage. Apart from increasing the heating power, the sensitivity can also be increased by designing the sensor structure with a high resonant frequency and this will be the focus of future research. Estimations based on equations (4) and (5) shows that the frequency-temperature sensitivity (based on $10 \mathrm{~V}$ heating) increases to $9 \mathrm{~Hz} / \mathrm{K}$ ( 30 times higher) as the resonant frequency increases to around $110 \mathrm{kHz}$. It is noted that to simplify the analysis, the stress-induced frequency shift is neglected, which results in a lower value of modelled sensitivity compared to the actual measurements described in the next section. Nevertheless, the presented analysis yields the modelled operation characteristics of the electrothermal resonant flow sensor.
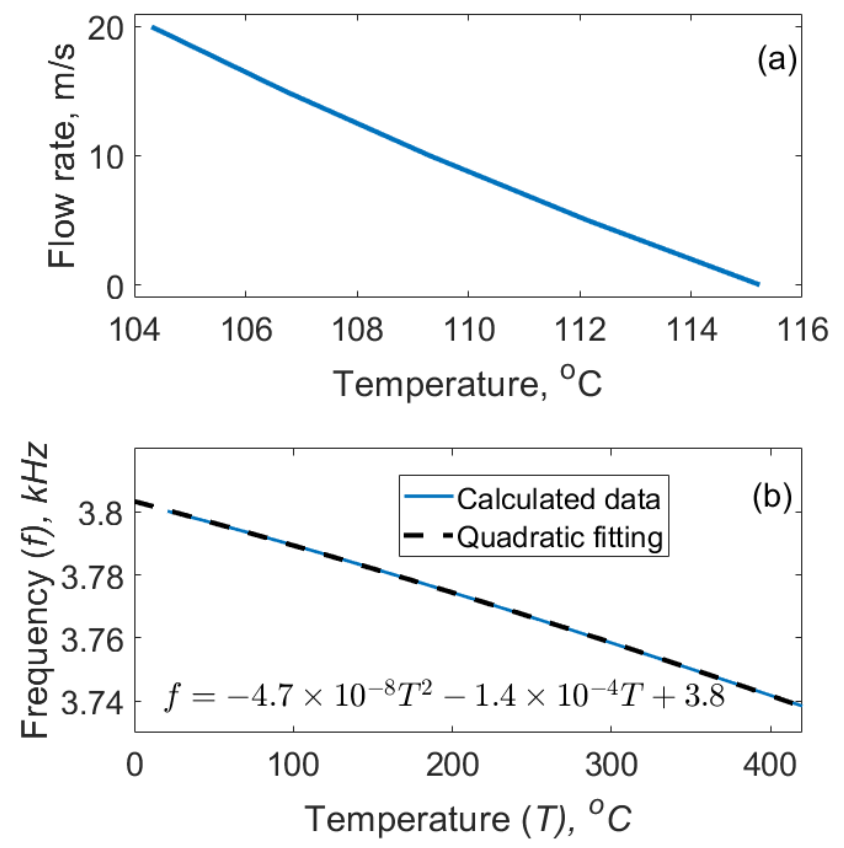

Fig. 5. (a) Calculated relation between the flow rate and average temperature for $5 \mathrm{~V}$ heating voltage. (b) Calculated resonant frequencies for a range of average temperatures.

In summary, an increase in velocity of a gas flow over the heated MEMS sensor cools the structure, and alters the Young's modulus, leading to variation of the resonance frequency. This is consistent with previous modelling and experiments on the temperature behavior of other MEMS cantilever structures [24], [25].

\section{EXPERIMENTAL EVALUATION OF MEMS GAS FLOW SENSOR}

An SEM image of the final fabricated silicon triple-beam cantilever sensor structure is shown in Figure 6.

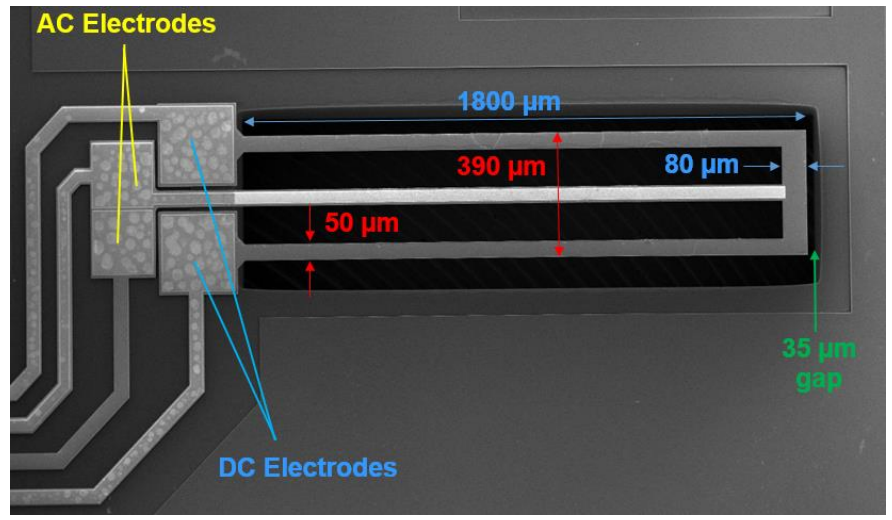

Fig. 6. SEM image of fabricated MEMS piezo-coated cantilever sensor.

Due to the small size of the fabricated sensor device, a noncontact method had to be employed for measuring the temperature of the cantilever structure and an infrared thermal imaging camera (FLIR SC7000 by FLIR Systems, Inc. USA) was used. Emissivity values of silicon can range from 0.4 to 0.7 dependent on parameters such as the emission wavelength of the radiation [26]. Hence, a series of experiments were conducted using the thermal camera to determine the temperature of test silicon wafers and a material of known emissivity upon a hot plate. The emissivity parameter was set to 0.5 in the camera control software for camera calibration as this was found to give a reliable correlation with the measured temperatures of the test silicon wafers. A DC voltage supply was connected to the outer folded-beam cantilevers and was used to heat the device. A thermal image of temperature distribution around the folded-beam cantilever for an applied voltage of $5 \mathrm{~V}$ (current $8.8 \mathrm{~mA}$ ) is shown in Figure 7 for zero gas flow velocity over the sensor. Similar thermal images were obtained for voltages ranging from $0 \mathrm{~V}$ DC to $5 \mathrm{~V}$ DC. The camera software was then used to obtain the direct graphical display of the temperature profile along the surfaces of the three-beam device. These profiles are shown in Figure 8 for the different DC voltages.

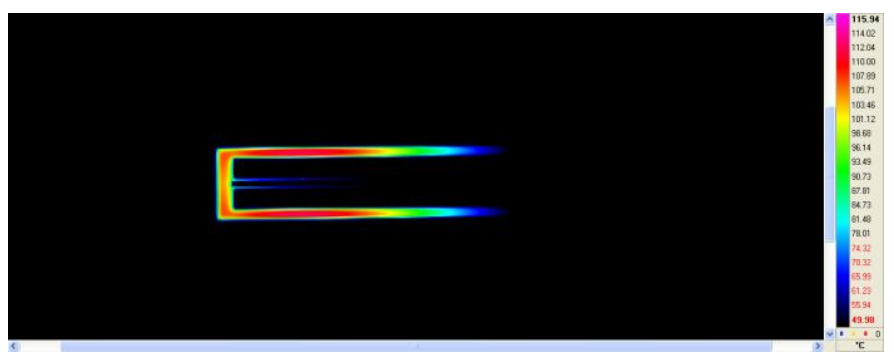

Fig. 7. Captured image of the measured temperature of the cantilevers for an applied voltage of $5 \mathrm{~V}$. 
These results and trends are consistent with our modelling conducted earlier, as shown in Figure 4(a) for zero gas flow velocity. It can be seen from the temperature profiles shown in Figure 8 that there are abrupt and spiked temperature steps at the center of the graph, in the region where the three cantilevers are linked together by the short connecting beam. This is thought ot be due to small fabricatiob and deposition irregularities at the junction of the 'hot' and 'cold' beams yielding irregularities in the thermal image of this zone.



Fig. 8. Change in measured temperature along the folded beams of cantilever device with applied DC voltage.

There is an observed difference between the measured temperature profile and that calculated by modelling, with the simulated profiles showing an elevated temperature. This may arise from several factors, such as the modelling neglects parasitic energy losses in the on-chip SOI/Al tracks; the accuracy of the thermal camera (the quoted manufacturers accuracy $\pm 2{ }^{\circ} \mathrm{C}$, the experimentally chosen emissivity, and the temperature profile area on the narrow cantilever images being smaller than that recommended); and manufacturing dimensional tolerances of the MEMS device. Considering the range of uncertainties, there is a good match between measurements and calculations.

Subsequently, a constant $5 \mathrm{~V}$ was applied to the outer beams and a regulated supply of nitrogen gas was flowed through a surrounding channel at different rates over the device to effect cooling of the structure, and the temperature along the folded beam was recorded for each nitrogen flow rate (Figure 9) using the thermal imager. The nitrogen flow rate was varied with a flowmeter (FGTF2BHD-L, Roxspur Measurement \& Control Ltd) over the range $0 \mathrm{ml} / \mathrm{min}$ to $5000 \mathrm{ml} / \mathrm{min}$.

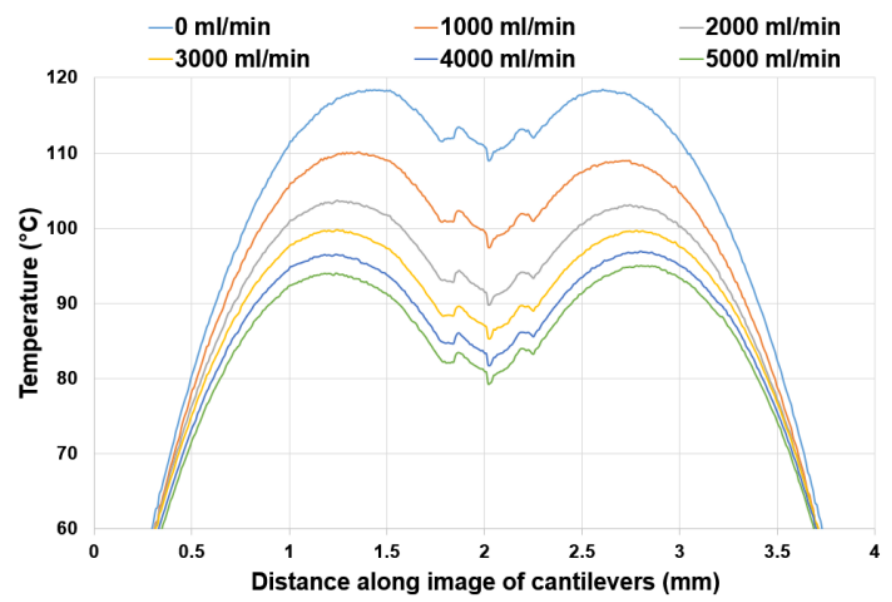

Fig. 9. Change in measured temperature of cantilever structure with changing nitrogen flow rate across the device.

Figure 10 is a plot of change in temperature with nitrogen flow rate of the highest temperature point of the folded beams. Again, the results validate those predicted by the mathematical modelling of the sensor.

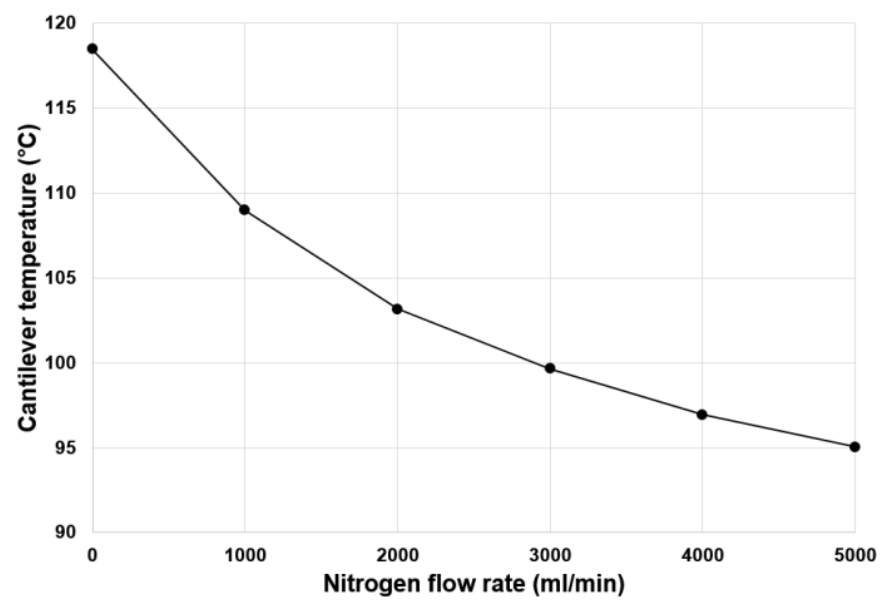

Fig. 10. Change in peak temperature of the folded beam due to changing gas flow rate over sensor.

Experiments were then conducted to investigate the effect of changing temperature on device resonance frequency. A DC voltage supply was connected to the folded cantilever to heat the device, and a function generator (TGA1241, TTi) was connected to the central piezoelectric-coated arm and was used to apply a sinusoidal voltage to this cantilever to set the device into oscillation. As stated earlier the combined silicon cantilevers and the piezoelectric coating form a triple-beam resonator sensor with natural frequency in the low $\mathrm{kHz}$ range. A schematic of the experimental setup is shown in Figure 11. The MEMS gas flow sensor was positioned inside a transparent test chamber beneath a laser Doppler vibrometer (Polytec OFV 512) which was used to measure the displacement of the cantilever resonator when it was in motion. The frequency at which maximum displacement occurred was taken as the 
resonance frequency. The resonance frequencies were determined with the laser spot focused at the center of the end section of the cantilever structure (as illustrated by inset to Figure 11).

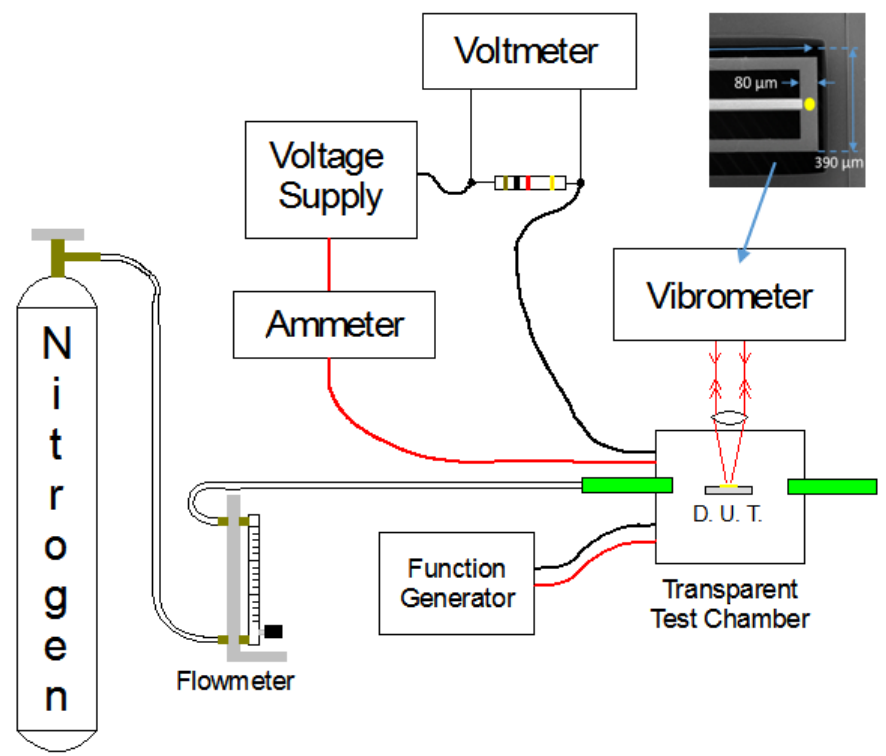

Fig. 11. Schematic of experimental setup shown the device under test (D.U.T.) within a test chamber and interrogated with a laser vibrometer. The yellow spot in insert indicates the laser spot position at the midpoint of the folded beams.

Under excitation of the central piezoelectric-coated arm by a $1 \mathrm{~V}_{\mathrm{pp}}$ sine wave, the first modal resonance was observed at $3.499 \mathrm{kHz}$, with higher order modes at $22.539 \mathrm{kHz}$ and 31.235 $\mathrm{kHz}$. These measured frequencies are close to those simulated for the MEMS structure as shown in Figure 2. Figure 12 shows the frequency domain of the resonance signal, which revealed a $3 \mathrm{~dB}$ bandwidth of $42 \mathrm{~Hz}$ and a quality factor of 83 .

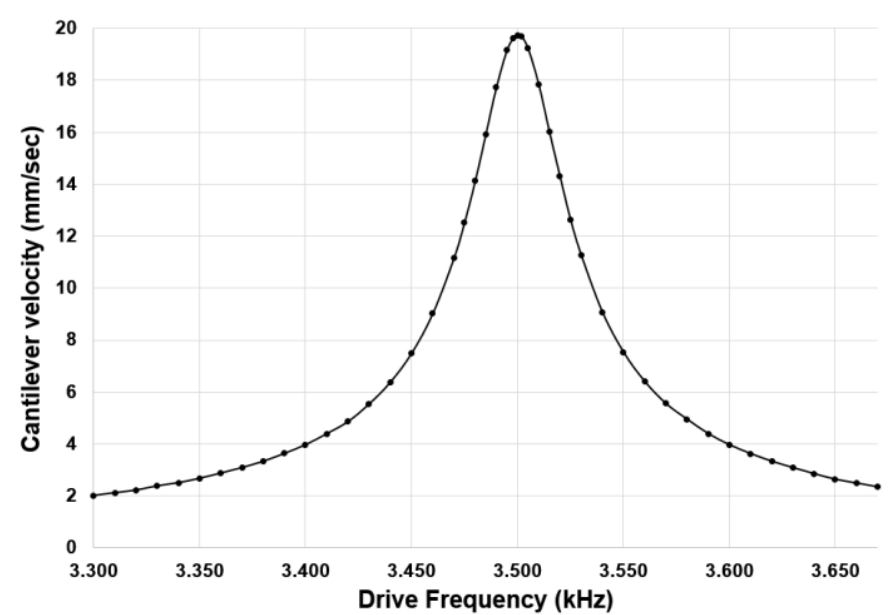

Fig. 12. Resonance versus frequency of drive voltage for cantilever sensor with no heating and no nitrogen flow.
A fixed heating voltage was applied to the folded beam and the MEMS device was set into resonance. This was repeated for different heating voltages, as well as no heating. Figure 13 shows the resulting decrease in measured resonance frequency (under zero gas flow conditions) with increasing heating, and this effect was fully reversible. This verifies the theoretical basis of the sensor, that the fundamental resonance of the device will be dependent on the temperature of the triple-beam cantilever structure.

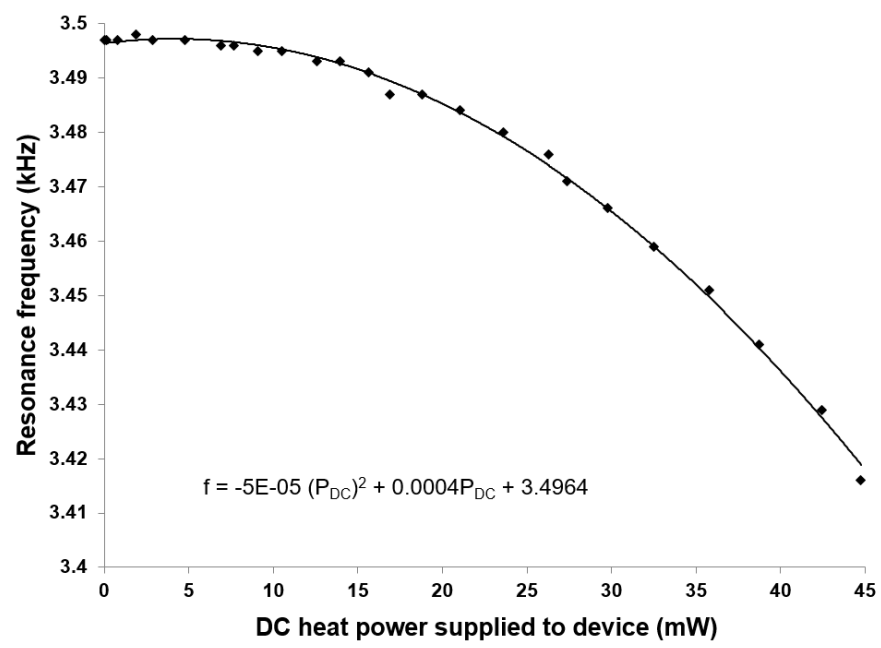

Fig. 13. Change in resonance frequency of MEMS cantilevers with increasing device heating (no gas flow over sensor). $\mathrm{P}_{\mathrm{DC}}$ denotes the DC power whilst $\mathrm{f}$ is the resonance frequency.

The next stage was to investigate if a changing gas flow over the heated triple-beam resonator would induce a change in temperature of the device, resulting in a measureable change in its resonance frequency. Regulated nitrogen gas flow was fed into the chamber and over the MEMS device at a variable flow rate. Figure 14 shows the change in resonance frequency with nitrogen flow rate for either no heating voltage or a range of fixed heating voltages. It can be seen that when the beam is not heated $(0 \mathrm{~V})$ no significant change in resonance frequency occurs with changing flow rate, confirming the change in resonance is produced only by a temperature reduction due to the gas flow. Thus for a fixed heating voltage the resonance frequency of the MEMS sensor can be directly calibrated with the rate of gas flow over the sensor. For an applied voltage of $5.1 \mathrm{~V}$ and using the fitted linear response in Figure 14 the sensor demonstrated a slope response of $66.7 \mathrm{ml} / \mathrm{min}$ per $\mathrm{Hz}$. 


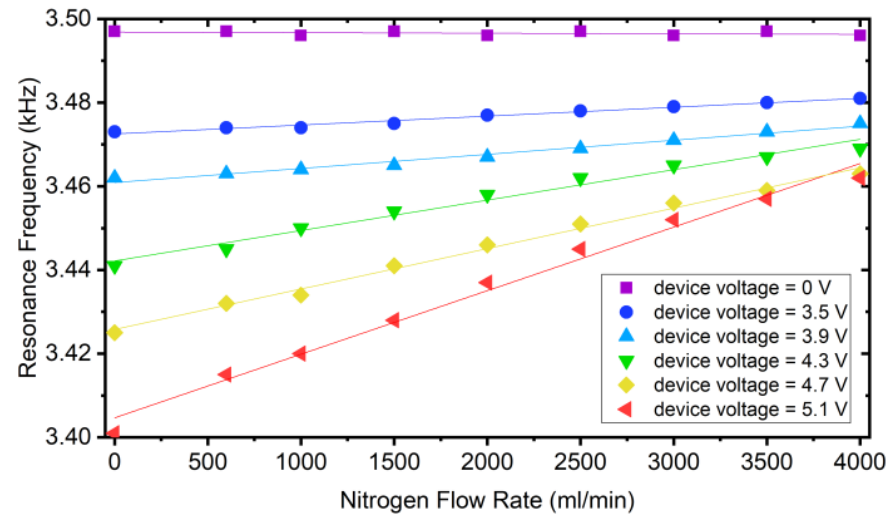

Fig. 14. Shift of fundamental resonance frequency of MEMS sensor with changing gas flow rate.

The response time was estimated by applying a step voltage change of the DC heating voltage to induce a step change in device temperature rather than a change in the nitrogen flow rate, since the changes in flow rates occurred over significantly longer time scales than the measured device response times. The 10 to $90 \%$ change response time for a 0 to 5 volts step was $47 \mathrm{~ms}$, whilst it was $15 \mathrm{~ms}$ for a 5 to 0 volts step change.

The stability of the sensor was investigated. Figure 15 shows a graph of the sensor with a constant heating voltage of either 5 or 4 volts and a constant $3000 \mathrm{ml} / \mathrm{min}$ nitrogen flow over it. The velocity of the cantilever was measured every 20 seconds over a period of one hour. Note, the $5 \mathrm{~V}$ stability curve was offset by $0.2 \mathrm{~V}$ for clarity of observation.

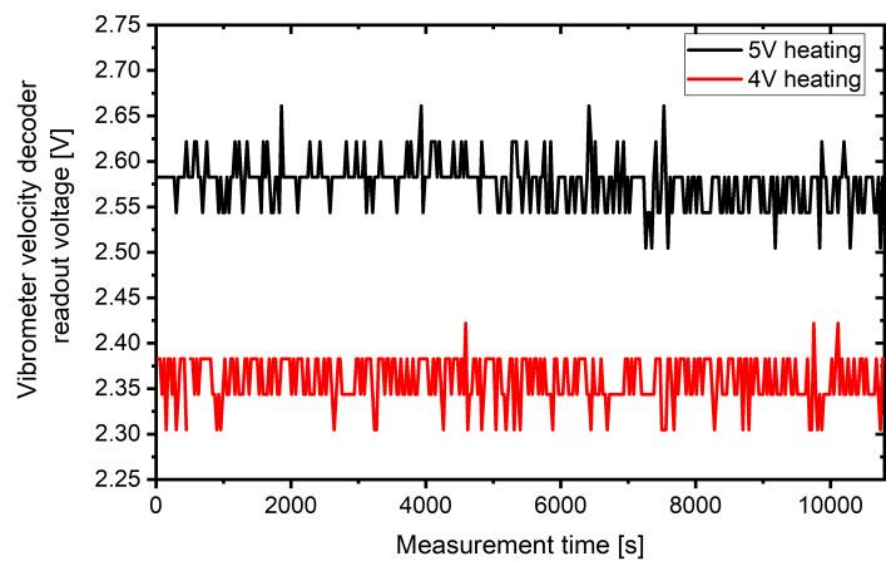

Fig. 15 Stability of cantilever sensor under constant nitrogen flow at different heating voltages.

These experiments showed there is no appreciable drift during this time period. In the case of a heating voltage of $5 \mathrm{~V}$ and $4 \mathrm{~V}$ respectively, if the outlier points on the graphs are ignored the velocity showed a variation of $\pm 0.8 \%$ in each case, whilst including the outlier points the velocity had a variation of \pm $3.2 \%$ and $\pm 1.6 \%$ respectively.

To demonstrate reversibility, a sensor had a fixed heating voltage of $5 \mathrm{~V}$ applied to it and the nitrogen flow over it was cycled fifty times between 0 and $4000 \mathrm{ml} / \mathrm{min}$ and 0 and 5000 $\mathrm{ml} / \mathrm{min}$. For the 0 and $4000 \mathrm{ml} / \mathrm{min}$ cycles, this gave a standard deviation (SD) of the higher frequency of $0.32 \mathrm{~Hz}$ (a variation from average of $\pm 0.02 \%$ ) and a SD of the lower frequency of $0.48 \mathrm{~Hz}$ (a variation from average of $\pm 0.03 \%$ ). For the 0 and $5000 \mathrm{ml} / \mathrm{min}$ cycles, this gave a SD of the higher frequency of $0.42 \mathrm{~Hz}$ (a variation from average of $\pm 0.04 \%$ ) and a SD of the lower frequency of $0.52 \mathrm{~Hz}$ (a variation from average of \pm $0.03 \%)$.

\section{CONCLUSION}

We have designed, simulated and fabricated a MEMS gas flow sensor based on the shift in resonance of a triple-beam cantilever structure. Mathematical modelling demonstrated that the fundamental resonance of the sensing device would change with changing gas flow rate due to the cooling effect of the gas. After fabrication of the device the piezoelectric coating on the central beam was actuated by an AC signal around $3.5 \mathrm{kHz}$ to drive the sensor into resonance while the folded beam of the sensor was heated with a DC voltage. The changing velocity of gas flow over the sensor modified the temperature of the device and induced a shift in the fundamental resonance frequency, experimentally verifying the modelling. By measuring the shift in resonance of the device, an anemometer function was demonstrated for gas flow rates up to $4000 \mathrm{ml} / \mathrm{min}$.

This novel MEMS device uses an outer U-shaped cantilever, which allows greater heat loss compared to a previously reported device design. In addition, it requires lower drive voltage to generate oscillations and uses aluminum nitride (AlN) as the piezoelectric material to generate oscillations. AlN has a higher electrical efficiency and greater compatibility with direct inclusion in MEMS fabrication processes compared with PZT, making it a more practical piezoelectric material in the MEMS context.

Future work will include the fabrication of an electrically isolated cantilever section coated with a piezoelectric material that itself could be used to sense the changes in the magnitude of the resonance peak (rather than using an external laser vibrometer) thus enabling on-chip electronic readout from a compact device. The integration of piezoelectric actuation and sensing elements on a silicon platform allows the potential for sensing to be carried out in a fully integrated electrical manner.

\section{REFERENCES}

[1] S. R. Karumuri, Y. Srinivas, J. V. Sekhar and K. G. Sravani, "Review on breakthrough MEMS technology," Arch. Phy. Res., vol. 2, no. 4, pp. 158165,2011

[2] H. Ma, Y. Du, M. Wei, E. Ding and L. Lin, "Silicon microheater based low-power full-range methane sensing device," Sensor. Actuat. A-Phys., vol. 295, pp. 70-74, 2019.

[3] F. Ejeian, S. Azadi, A. Razmjou, Y. Orooji, A. Kottapalli, M. E. Warkiani and M. Asadnia, "Design and applications of MEMS flow sensors: A review," Sensor Actuat. A-Phys., vol. 295, pp. 483-502, 2019.

[4] Y. Kamada, A. Isobe, T. Oshima, Y. Furubayashi, T. Ido and T. Sekiguchi, "Capacitive MEMS accelerometer with perforated and electrically separated mass structure for low noise and low power," $J$. Microelectromech. S., vol. 28, no. 3, pp. 401-408, June 2019. 
[5] R. Abdolvand, B. Bahreyni, J. Lee and F. Nabki, "Micromachined resonators: A review," Micromachines, vol. 7, no. 9, pp. 160-216, 2016.

[6] H. Yunhong, Z. Meng, H. Guowei, S. Chaowei, Z. Yongmei and N. Jin, "A review: aluminum nitride MEMS contour-mode resonator," $J$. Semicond., vol 37, no. 10, pp. 101001-101010, 2016.

[7] A. Uranga, J. Verd and N. Barniol, "CMOS-MEMS resonators: From devices to applications," Microelectron. Eng., vol. 132, pp. 58-73, 2015.

[8] D. Mulik, G. S. Phadke and S. Salunkhe, "Comparative study of conventional and MEMS flow meters," IJCA Proceedings on International Conference on Computer Technology, no. 3, pp. 32-37, 2015.

[9] M. Marraccini, K. Bak-Kristensen, A. Horn, E. Fifield and S. O. Hansen, "Influence of small-scale turbulence on cup anemometer calibrations," $J$. Phys.: Conf. Ser., Vol. 926, p. 012005, 2017.

[10] R. Y. Que, R. Zhu, Q. Z. Wei and Z. Cao, "Temperature compensation for thermal anemometers using temperature sensors independent of flow sensors," Meas. Sci. Technol., vol. 22, no. 8, p. 085404, 2011.

[11] K. Naeli and O. Brand, "Dimensional considerations in achieving large quality factors for resonant silicon cantilevers in air," J. Appl. Phys., vol. 105, no. 1. p. 014908, 2009.

[12] N. Lavrik, M. J. Sepaniak and P. G. Datskos, "Cantilever transducers as a platform for chemical and biological sensors," Rev. Sci. Instrum., vol. 75, no. 7, pp. 2229-2253, 2004.

[13] Y. J. Rao, D. Walsh, D. Uttamchandani and B. Culshaw, "Temperature dependence of resonant frequency in all-fibre optically addressed silicon microresonator sensors," Electron. Lett., vol. 27, no. 11, pp. 934-935, 1991.

[14] S. Bouwstra, R. Legtenberg, H. A. C. Tilmans and M. Elwenspoek, "Resonating microbridge mass flow sensor," Sensor. Actuator., vol. A21A23, pp. 332-335, 1990.

[15] Y-H. Wang, C-Y. Lee and C-M. Chiang, "MEMS-based air flow sensor with a free-standing microcantilever structure," Sensors, vol. 7, pp. 23892401, 2007.

[16] Y-H. Wang, C-P. Cheng, C-M. Chang, C-P. Lin, C-H. Lin, L-M. Fu and C-Y. Lee, "MEMS-based gas flow sensors," Microfluid. Nanofluid., vol. 6, pp. 333-346, 2009

[17] L. Li, V. Stankovic, L. Stankovic, L. Li, S. Cheng and D. Uttamchandani, "Single pixel optical imaging using a scanning MEMS mirror". $J$. Micromech. Microeng., vol. 21, p. 025022, 2011.

[18] A. Paterson, R. Bauer, W. Lubeigt, and D. Uttamchandani, "Tunable Yb:KGW Laser, CW or Q-switched, enabled by dual-axis tilt of an MOEMS Mirror," IEEE J. Sel. Top. Quantum Electron., vol. 24, no. 5, p. $1601709,2018$.

[19] L. Li and D. Uttamchandani, "Dynamic response modelling and characterization of a vertical electrothermal actuator," J. Micromech. Microeng., vol. 19, p. 075014, 2009.

[20] S. Tsutomu, "Spectral emissivity of silicon," Jpn. J. Appl. Phys., vol. 6, p. $339,1967$.

[21] E. Sartori, "Convection coefficient equations for forced air flow over flat surfaces," Sol. Energy, vol. 80, pp. 1063-1071, 2006.

[22] U. Gysin, S. Rast, P. Ruff and E. Meyer, "Temperature dependence of the force sensitivity of silicon cantilevers," Phys. Rev. B, vol. 69, no. 4, p. 045403, 2004.

[23] R. Sandberg, W. E. Svendsen, K. Molhave and A. Boisen, "Temperature and pressure dependence of resonance in multi-layer microcantilevers," J. Micromech. Microeng., vol. 15, no. 8, p. 1454-1458, 2005.

[24] E. Iervolino, M. Riccio, A.W. van Herwaarden, A. Irace, G. Breglio, W. van der Vlist and P.M. Sarro, "Temperature dependence of the resonance frequency of thermogravimetric devices," Procedia Eng., vol. 5 pp. 948951,2010

[25] M. Stifter, H. Steiner, T. Sauter, F. Keplinger and W. Hortschitz, "Thermal influences on driven damped MEMS cantilevers," IEEE Microelectronic Systems Symposium (MESS), 8-9 May, Vienna, Austria. pp. 1-5, 2014.

[26] N. M. Ravindra, B. Sopori, O. H. Gokce, S. X. Cheng, A. Shenoy, L. Jin, S. Abedrabbo W. Chen and Y. Zhang, "Emissivity measurements and modeling of silicon-related materials: An overview," Int. J. Thermophys., vol. 22, no. 5, pp. 1593-1611, 2001.

Robert Blue received the Ph.D. degree in Optical Electronics from the University of Strathclyde, Glasgow, U.K., in 1996. He is currently at the Centre for Microsystems and Photonics, University of Strathclyde. His research interests include MEMS-based wavelength selective devices, the development of MEMS explosive sensors incorporating novel nitro-sensitive polymers, and laser generated ultrasound for imaging tissue.

James G Brown received a B.Sc. degree in physics and electronic engineering from the University of Glasgow, Glasgow, UK in 1985 and an M.Sc. in laser engineering from St Andrews University, St Andrews, UK in 1995. Since 1999 he has been working in research in MEMS at the University of Strathclyde, Glasgow, UK, focusing on metrology of MEMS devices and applications of MEMS scanners.

Lijie Li (SM'10) received the Ph.D. degree from University of Strathclyde, Glasgow, U.K., in the area of MEMS, in 2004. He is professor at the College of Engineering, Swansea University, United Kingdom. His research interests are in developing MEMS transducers, energy harvesting devices, piezotronics, optical and radio frequency (RF) MEMS devices and systems. Moreover, he is interested in first principle study of nanodevices and nanomaterials, as well as machine learning techniques with applications in transducers. He is Fellow of IET, and senior member of IEEE.

Ralf Bauer (M'17) received the Dipl.Ing. degree in mechatronics from the University of Erlangen-Nuernberg, Germany, in 2010, and the Ph.D. degree from the University of Strathclyde, Glasgow, U.K., in 2013, for work on MEMS enabled solid-state lasers.

$\mathrm{He}$ is currently a Lecturer and RAEng Engineering for Development Research Fellow in the Department of Electronic and Electrical Engineering at the University of Strathclyde, Glasgow. His research interests are in the field of MEMS enabled sensors and systems in the area of biomedical sensors, optical systems and biomedical imaging systems.

Deepak Uttamchandani (SM'05, F'19) received the Ph.D. degree from University College London, London, U.K., in the area of optical fiber sensors, in 1985. His early research in MEMS concentrated on optothermal microresonator sensors and in investigating techniques for general MEMS material characterization using micromechanical resonators. His recent research has concentrated on developing system applications of optical MEMS such as intracavity MEMS-based laser systems, MEMS-based photoacoustic spectroscopy for gas sensing, and MEMS-based single-pixel imaging systems. He has also published in the field of sub-wavelength tip-based Raman spectroscopy, which has contributed to the development of tipenhanced Raman spectroscopy, and in the area of in situ, intraocular drug detection systems via optical spectroscopy in the living eye. 